\section{Professional standards and their escalating impact upon the dental profession}

\author{
K. Lewis ${ }^{1}$
}

IN BRIEF

- Explains the legal background to the professional standards intended to be applicable to UK dentistry.

- Explores the mechanisms through which misunderstandings and misinterpretation have arisen.

- Suggests that the wrong standards are being used and applied by experts and those involved in GDC fitness-to-practise procedures.

This article highlights several areas of confusion that have arisen in relation to professional standards in the UK. In doing so, it provides a possible explanation for the fact that litigation levels against UK dentists, and also the level of regulatory challenge and criticism that they face, are both at levels far beyond those that are seen in virtually any other country in the world.

\section{BACKGROUND}

In almost every arena in which Dental Protection assists dentists, the question of professional standards arises - for example, whether or not the standard of care and treatment, facilities, record keeping or professional conduct (or whatever) is satisfactory.

Dental Protection is increasingly of the view that expectations of UK dental health professionals have become unrealistic and unsustainable, and too many bodies and individuals within them, and/or working on their behalf, are competing with each other to raise the bar ever higher without pausing to think much about what they are doing and why.

\section{CLINICAL NEGLIGENCE AND THE BOLAM STANDARD}

The time-honoured Bolam ${ }^{1}$ principle - even though the case in question dates from over half a century ago - still applies, in that a practitioner is not negligent if $s /$ he has acted in a manner which is considered to be appropriate by a responsible body of opinion among people working in the same field, and professing to have the same skills as the practitioner in question.

Many years later, the Bolitho ${ }^{2}$ judgement made it clear that a clinician's failure to follow a practice which the majority 'reasonable body of opinion' might consider appropriate, does not necessarily mean that

'Dental Director, Dental Protection Ltd, 33 Cavendish Square, London, W1M OPS

Correspondence to: Mr Kevin Lewis

Email:Kevin.Lewis@dentalprotection.org

\section{Refereed Paper}

Accepted 18 March 2015

DOI: $10.1038 /$ sj.bdj.2015.251

${ }^{\circ}$ British Dental Journal 2015; 218: 381-383

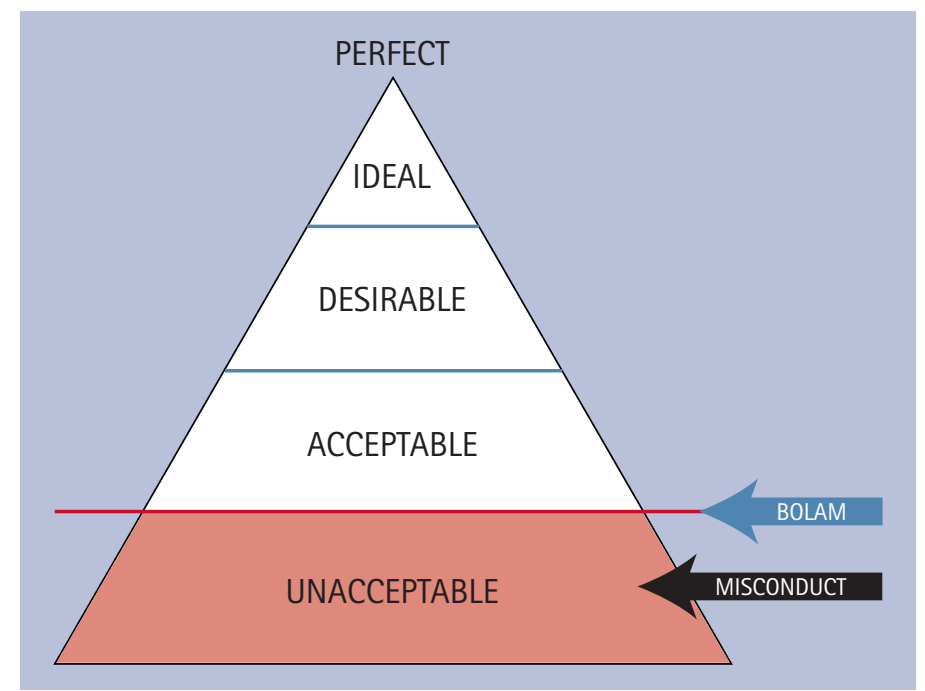

Fig. 1 The intention of legal standards the clinician has acted negligently. The practitioner's defence in law in such a situation is that although s/he has followed a minority alternative body of opinion, that opinion is both logical and responsible.

The challenge, of course, is to persuade a court of law that the body of opinion which you are following is not only responsible, but also respectable, reasonable, logical and (when the context demands) seeks an appropriate balance between potential risks and benefits.

The 'no win - no fee' law firms have developed a panel of experts upon whose services they regularly call. While they have a duty to serve the court, rather than the solicitors who are instructing them, the regularity of their instructions reflects their willingness and propensity to set the bar (of what is and is not an appropriate standard) at a level above that of a reasonable clinician in the same field.

There is a need to ensure that the clinician is measured against a reasonable peer group, rather than against the standard of a specialist carrying out the same procedure(s).

Part of the problem is the baseline standards against which the registrants are being measured, as it is easier to fall seriously short of a standard if that standard is being placed at an unreasonably high level in the first instance. It is only by falling 'seriously' short of a reasonable professional standard that a finding of 'misconduct' should ever be made by one of the GDC's fitness to practise committees.

In Figure 1, the line separating 'acceptable' from 'unacceptable' represents the 'reasonable' standard established by the Bolam principle. The current regulatory standard of 'misconduct' should represent a serious departure from such a 'reasonable' standard.

By misinterpreting aspirational guidelines as the 'reasonable' standard that equates to the intention of Bolam, a much larger range of performance that would previously have represented an 'acceptable' or even 'desirable' standard, appears to fall below that 
elevated required standard (Fig. 2). This in turn allows a finding of 'misconduct' to be made by the GDC in situations where most registrants would find the achieved standard perfectly acceptable or even better than that.

\section{THE DENTO-LEGAL STATUS AND PITFALLS OF GUIDELINES AND PROTOCOLS}

Hardly a week passes in our busy professional lives without some organisation, 'official' body or special interest group issuing a new set of guidelines or protocols for members of the dental profession to follow. Most of the time these documents tend to be aspirational in nature - that is, they are produced in good faith and with the best of intentions to suggest some kind of desirable standard of what could (or in some instances, should) be achieved. Sometimes there is a special reason or a vested interest to explain why the guidelines are being produced at a particular time, or in a particular form, and/ or why any desired standard described in the guidelines is set at a particular level.

\section{LEGAL STATUS}

A useful starting point is to consider the extent to which guidelines and protocols carry any legal status and in the light of this, what kind of weight can be attached to them. Any 'best practice' document may well set the bar (of what is and is not acceptable) at a level which greatly exceeds the reasonable and appropriate standard that the law expects of us.

It is interesting that in the USA for example, guidelines and protocols are treated by the courts as 'hearsay' evidence, with a consequential downgrading of their evidential weight.

This is an entirely logical perspective because unless, and until, the court can hear evidence from (perhaps) the author(s) of the guidelines that their evidence can be rigorously cross-examined and any objective scientific basis for the guidelines scrutinised, then there is no way of establishing whether or not the opinion satisfies the criteria set out above.

Published guidelines and protocols are often referred to in courts of law or in hearings before regulatory bodies such as the GDC, yet very often nobody knows for sure how the guidelines came to be written and adopted (or by whom) and nobody knows how vigorously they were challenged before publication. The more sets of guidelines there are in circulation, the more problematic the picture becomes.

\section{FACTS VERSUS OPINION}

The ground covered in some sets of guidelines

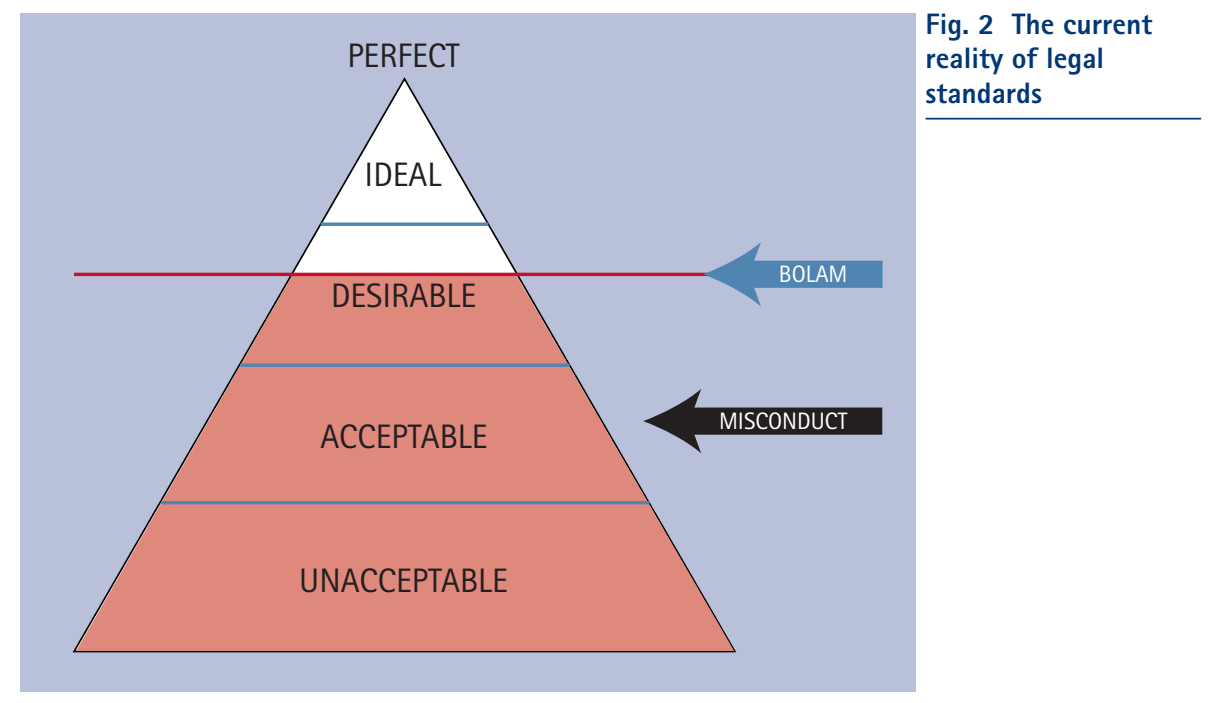

will touch upon matters of law ie situations in which specific legislation exist to point the way. Here, the requirement to comply with the stated standard will be absolute and unequivocal. It is unhelpful, therefore, when guidelines contain factual matter of this nature interspersed with other matters of subjective opinion, without making a clear distinction between the two or (worse still) by wrongly implying that the legal requirement extends to all aspects of the guidelines.

The average reader may find it extremely difficult to separate the two and may, as a result, place too little weight on definitive legal requirements and perhaps too much weight on suggestions that amount to little more than the point of view of the author(s).

\section{CONFLICTING GUIDELINES}

It is not unusual for there to be more than one responsible body of opinion, represented within diametrically opposing guidelines. The recurring debate over antibiotic prophylaxis is one such example, and even now there are pockets of very authoritative (and outspoken) opinion which are quite different from the 'mainstream' opinion published by NICE, endorsed by the Department of Health and published in the Dental Practitioners Formulary.

\section{CHANGING GUIDELINES}

An inevitable consequence of any guidelines published in hard copy is that one set of guidelines will be superseded by another either as a direct like-for-like replacement, or because one set of guidelines acquires some degree of pre-eminence over another from a different source.

You may be following the guidelines issued by an employer, organisation or association of which you are a member, but these guidelines may set the bar higher or lower than an alternative set of guidelines from another source. The fact that one set of guidelines pre-dates another may or may not be relevant, although it might appear logical to assume that the most recently issued guidelines will be the most up-to-date with current thinking. This is not always the case.

One advantage of publishing guidelines on the Internet is that they can easily be updated whenever necessary. Sometimes, outdated guidelines are not replaced because some further clarification or development is being awaited before finalising the text of any replacement document this was the case with the decontamination guidelines HTM 01-05 for several years. This can be a dangerous and misleading time for anyone who is making important decisions based upon the guidelines available at the time - for example, someone contemplating a significant capital investment (eg decontamination equipment) that is keen to ensure their choice of equipment is not rendered obsolete almost as soon as it is installed.

\section{LEVEL OF AUTHORITY}

A further problem for practitioners is that not all sets of guidelines will carry the same degree of authority. Some are likely to be viewed as highly authoritative and good reasons would then need to be given for departing from them. Other guidelines represent little more than the consensus point of view of a few individuals who happened to be given the task of producing the guidelines. The difficulty for the busy practitioner is that of deciding how to distinguish between these two extremes as the context is very rarely made clear when guidelines are published.

It is not uncommon for a group of eminent experts in a field to be brought together and their collective wisdom brought to bear upon a particular topic. Any resulting standards document, protocol or guidelines will have enormous authority and credibility and may well be relied upon by bodies such as 
the GDC. But the very expert status of the authors may be self-defeating in the context of a negligence claim, because it may not represent the reasonable standard expected of the 'ordinary competent person skilled in that particular art' as described in Bolam. Not surprisingly, claimant lawyers will seize upon whichever set of guidelines best suits the cause of their client.

\section{EVIDENCE BASE}

Yet another difficulty that besets guidelines and protocols is that - even within the same set of guidelines - the text alternates between objective, evidence-based fact and entirely subjective opinion.

There is an ever-present danger that such opinion will be subsequently quoted by others as if it were incontrovertible fact. By that stage nobody tends to question the source or scientific basis for the statement, nor stops to consider whether or not the statement is still being made in its original context. One such example was that of the purported 'requirement' to record the batch number of local anaesthetic solutions administered in dentistry. Not only is this bizarre expectation starting to appear in checklists used by primary care trusts and others, as well as in guidance and 'best practice' documents on record keeping, it has even appeared as one of the charges in more than one GDC fitness to practise hearing before the Professional Conduct Committee.

Dental Protection has been unable to identify a single case where the batch number of a local anaesthetic solution has become an issue of any kind in terms of patient safety or an adverse event, although many confused dental members (including senior academics) have contacted us to question why they are being criticised by third parties for not recording these details.

In the view of Dental Protection, such requirements are not only unnecessary and unscientific but are also directly contributing to the problems frequently seen in clinical record keeping. While practitioners are being coerced into recording peripheral details such as the batch numbers of local anaesthetic solutions, they are squandering precious time that could and should be put to much better use - either in communicating with patients or in recording more significant details of the treatment provided.

The perversity of this example of illconceived guidelines is that the same 'requirement' to record batch numbers is not extended to the many other pharmacological agents and materials that are used in everyday dentistry - from distilled water to sodium hypochlorite and the endless range of dental materials. The suggestion simply does not withstand logical scrutiny.

\section{TERMS OF REFERENCE}

When reading any new set of guidelines or protocols, it is important to understand at the outset whether these are intended as 'minimum standard' guidelines, aspirational guidelines of 'desirable' standards or 'best practice' guidelines. If the latter, is the suggested standard one that is applicable today, or has it been deliberately set at a more challenging level for practitioners to move towards over a period of time?

HTM 01-05 was an excellent example of getting this right, when a clear distinction was drawn between the minimum standard at a defined moment in time, and a more aspirational 'direction of travel' beyond that.

An accurate description of context is important, not just in practical terms but also in a dento-legal sense. Yet this is rarely made clear, and authors of guidelines would assist readers by addressing any such ambiguity.

\section{CONFLICTS}

Guidelines and protocols are being produced in unprecedented numbers. In one sense this is helpful and desirable, but it can lead to information overload and as a result, a studied apathy and inactivity.

This is made more likely when two or more sets of guidelines are issuing conflicting advice and the practitioner is understandably confused as to which set of guidelines to follow. When these bodies appear to be of similar standing and authority, these difficulties are heightened.
But the peril here is the fact that some guidelines carry much greater weight in one area of practice than in another. The NICE guidelines on dental recall intervals would be one such example; following them is a requirement of the NHS (General Dental Services) Contracts Regulations $2005^{3}$ and the NHS (Personal Dental Services) Agreements Regulations 2005, ${ }^{4}$ and a condition of the provider contracts made under them.

The NICE guidelines may only be recommendations, but they gain additional weight when incorporated into legislation and legally-binding contracts. Outside the NHS they lose much of their authority because they have no more status than that of a single responsible body of opinion which needs to be set against other responsible bodies of opinion, in the eyes of the law.

\section{SUMMARY}

Dental Protection is acutely aware of the difficulties and dento-legal threats that guidelines can create, because of the large numbers of requests for advice that we receive in relation to various guidelines.

The authors of such guidelines could do a lot more to help the practitioner to navigate safely through the plethora of guidance impacting upon modern dentistry. In the meantime, a practitioner is expected to stay abreast of current thinking, to carefully consider all relevant guidelines and to act reasonably and responsibly in the light of them.

Ultimately, a clinician must make a responsible and informed judgement in the best interests of his/her patients, as well as being able to demonstrate that there were sound and valid reasons for acting in a particular way.

1. Bolam v Friern Hospital Management Committee (1957) 2 All ER 118; (1957) 1 WLR 582.

2. Bolitho $v$ City and Hackney Health Authority (1997) 39 BMLR 1; (1998) 1 Lloyds Rep Med 26

3. NHS England. NHS (general dental services) contracts regulations 2005. 2005. Online information available at http://www.legislation.gov.uk/ uksi/2005/3361/regulation/22/made (accessed March 2015).

4. NHS England. NHS (personal dental services) agreements regulations 2005. 2005. Online information available at http://www.legislation.gov.uk/ uksi/2005/3373/note/made (accessed March 2015). 